\title{
Toward a Modeling Framework for Organizational Competency
}

\author{
Reza Vatankhah Barenji ${ }^{1}$, Majid Hashemipour ${ }^{1}$, and David A. Guerra-Zubiaga ${ }^{2}$ \\ ${ }^{1}$ Department of Mechanical Engineering, Eastern Mediterranean University, Famagusta, \\ KKTC, Via 10 Mersin, Turkey \\ Reza.vatankhah@cc.emu.edu.tr \\ ${ }^{2}$ CIDESI Texas, 1700 Research Parkway, Suite 170, College Station, Texas, 77845, USA
}

\begin{abstract}
Competency modeling framework serves as a; (a) very important basis for the explanation of a generic competency modeling approach, (b) base element in the consolidation of existing knowledge in this area, (c) tool for model developers on selecting appropriate competency models, and (d) basis for competency modeling. This research uses literature review approach to propose a modeling framework for organizational competency. The proposed modeling framework has been developed based on the most relevant well known competency models. The research suggests that organizational competency can be categorized into three groups; individual competency, enterprise competency and collaboration-oriented competency. For modeling each of these groups, it is essential that the modeling process have to be aligned with model developer purpose (Modeling perspective), thus the model developing process will be based on the same segmentation model. Furthermore, competencies have to be model at different levels of abstraction (modeling intent).
\end{abstract}

Keywords: Competency, Capability, Modeling, Modeling framework.

\section{Introduction}

In early 1990's [1] described core competences as the "collective learning in the organization, especially how to coordinate diverse production skills and integrate multiple streams of technologies". There exist several definitions for competency. Notable among them, [2] defines competency as "the interaction between three components: the professional situations, the actors, and the resources" and, [3] describes competency as "the smallest autonomous performance unit able to create value, be indivisible and able to exist independently".

Competency model is an information and knowledge model that describes the skills and abilities of a particular organization. Organizations need comprehensive competency model for successful management of internal resources/activities and corresponding their inter-related activities [4]. For an organization to participate in Virtual Organization Breeding Environment (VBE) activities, prior submission of competency model is necessary [5]. On the other hand, competency models are 
essential tool for improving organizational core competency [6]. In small and medium size enterprises, the competency models can be developed from oral information while, in a more complex organization, the collection and modeling of competency by human actor is not any more effective [7]. In such cases, computer-based mechanisms are required. Available Literature reviewed emphasizes competency model as a paradigm which depend on modeling purpose that varies from one model to another [8]. Furthermore, collection, analysis and management of competencies for modeling purpose are a complex task involving many aspects of manufacturing and business environment.

These issues motivate the co-authors of this paper to propose a $\mathrm{PhD}$ thesis to the author. The main aims of this research thesis are: (a) understand capability and competency concepts (b) introduce an approach to store, manage and maintenance capability and competency of an organization in different levels of abstraction (c) suggest some criteria for using competency as a tool for organization integration. The central research questions which are addressed in this research thesis;

$R Q 1)$ how to model an organization with its existing competencies?

$R Q 2)$ what are the templates, procedures and methods to store, maintain and manage competency of an organization?

To answer the above research questions, the hypothesis prepared are as follows;

Hypothesis1) if we combine a multi-phase holonic modeling view with associated needed competencies then we can better understand product evolution, customization potential, and retrofitting needs.

Hypothesis1)If we combine competency model paradigm with co-innovation and involvement of local stakeholders then we can have a more effective life-cycle support for complex products, including customization, maintenance, and retrofitting. At the first step of the research thesis, based on existing well-known competency models, a conceptual competency modeling framework for organizational competency is developed. This step will be presented in this paper. For the next step, using a case study, a capability model of a shop is developed. The case study uses Flexible Manufacturing Systems (FMS) Lab of the Eastern Mediterranean University. Finally with the use of another case study, the enterprise competency model is proposed [9]. The case study was a bicycle manufacturing company which is located in Cyprus.

"A modeling framework can be seen as an envelope that includes a number of models, collections of templates, procedures and methods, rules, and even tools" [10]. Competency modeling framework is an important basis towards the development of competency models. Competency modeling framework intend to help model developers to identify what kinds of competency models have to be created for successful decision making [11]. What have not been published in competency modeling context, however, are, a comprehensive modeling framework for organizational competencies and, a generic capability and competency model which can be used by researchers and decision makers. The purpose of this paper is to develop a modeling framework for organizational competency. The modeling framework has been developed using a literature reviewed approach. In essence, the 
proposed modeling framework has been developed based on the literature review and evaluated with the aid of most relevant well known competency models.

\subsection{Contribution of the Paper to "Internet of Things"}

Competency modeling is an approach for configuring an organizations data base scheme. In contrast to the other organizational data modeling approaches that covers only the information and knowledge of the organization from one perspective, competency modeling has the capability of providing an organizational data model from different viewpoints. Similar to the other organizational data models, it is accessible through the internet using some specific infrastructures and tools, in which, all the tangible and intangible objects of the organization (such as, machines and processes) can be individually identified and presented in virtual environment. Since, "uniquely identifying objects and their virtual representations in an inter-like structure referred as "Internet of things"", the authors believe is that, competency modeling approach, is a good starting point on applying the "Internet of things" philosophy on organizational management context.

\subsection{Research Methodology}

To effectively conduct the proposed research investigation and develop a competency modeling framework (CMF) a research methodology developed as below. The proposed methodology illustrates the CMF configuration processes from requirements analysis (literature review approach) to $\mathrm{CMF}$ development. In the requirements analysis phase, primary 86 related journal papers were extracted both from computer science and organizational management data bases. After elimination of the papers with less novelty and or fewer citations, 46 papers have been used in the final requirement analysis phase. These requirements are then utilized for generalization phase which proposed the modeling framework perspectives. Ultimately, the generalized perspectives are employed to propose a competency modeling framework.

\section{Literature Review}

In this section, for discovering competency modeling framework perspectives, the most cited competency models are taken in to consideration. To begin with, the most relevant well known competency models on both organizational and computer science literatures are reviewed. After that, the potential perspectives for competency modeling framework are highlighted.

When attempting to establish a modeling framework, it is important to consider the potential inputs and partial contributions from previous related works for proper system requirements [12]. This contribution uses literature review approach for the requirement analysis phase. In this way, we strived to harness the advantages offered by a number of well-known competency models introduced by other initiatives, and attempted to eliminate some of the common pitfalls which theoretically or empirically, model developers face while establishing a model. 
Clearly, this attempt does not argue about the relevance and appropriateness of previous well known models, for the purpose of organizational competency modeling. Rather it argues and represents (i.e. through identifying more elements) that more needs to be done for the purpose of proper organizational competency modeling. As such, instead of starting from scratch to identify the main perspectives and required elements for competency modeling, we have tried as much as possible to reuse some already defined concepts that are more familiar to the users of model, model developers and researchers in this area. As an illustration, table 1 summarizes our analysis results from some well-known competency models for highlighting the potential perspectives for competency modeling framework at intra- enterprise.

Table 1. An attempt to map current competency models applicable to competency modeling framework

\begin{tabular}{|c|c|c|c|}
\hline Intra & REF. & RESEARCH CONTRIBUTION & MODELING AREA \\
\hline \multirow{4}{*}{ 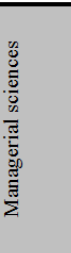 } & [1] & Core competency notation & $\begin{array}{l}\text { Organization competency, definition } \\
\text { at concept level }\end{array}$ \\
\hline & $\begin{array}{l}{[13]} \\
{[14]}\end{array}$ & Core competency hierarchy & $\begin{array}{l}\text { Organization competency, concept } \\
\text { model }\end{array}$ \\
\hline & [15] & $\begin{array}{l}\text { "To assist in core competence management an enabling core } \\
\text { competence lens model was presented together with a framework } \\
\text { for core competence maintenance" }\end{array}$ & $\begin{array}{l}\text { Enterprise competency, organization } \\
\text { competency, at detail level }\end{array}$ \\
\hline & [7] & $\begin{array}{l}\text { "Investigating the existence and nature of core competency } \\
\text { concepts within a section of UK SME manufacturing } \\
\text { organizations" }\end{array}$ & $\begin{array}{l}\text { Organization competency, concept } \\
\text { and basic level }\end{array}$ \\
\hline \multirow{2}{*}{ 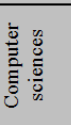 } & [16] & $\begin{array}{l}\text { Analyze IS role in raising organizational competencies and } \\
\text { prompting the cross-functional integration necessary to achieve } \\
\text { scale, scope, and learning curve economies for an enterprise. }\end{array}$ & $\begin{array}{l}\text { Organization competency at concept } \\
\text { level }\end{array}$ \\
\hline & [17] & Develop a framework for enterprise competency modeling & $\begin{array}{l}\text { Enterprise competency, organization } \\
\text { competency at concept level }\end{array}$ \\
\hline
\end{tabular}

A summary of our main observations are as follow:

- Similar to organization management theory, which hierarchically subdivides organization into four tiers; shop, enterprise, organization and network, the competency models depending on the organizational level differ from one tier to another. Shops as an organization just comprises of capability not competency [13] so there is no competency model for shop level. For enterprise level, there exist three different sets of competencies models namely individual, enterprise and collaboration-oriented. For organization level the models refers to core competencies of the organization and for network level the models deals with sets of competencies which each organization shared within the network.

- The reviewing on competency model literatures emphasis that competency model is a research context from both organizational management and computer science points of view meanwhile, this context is significant at intra-organization, interorganization and network levels.

- An important task during competency modeling is to provide an abstract representation of the model on which the model is to be used. The structure and level of details depending on the further intentions of using this specific model is a three level architecture: concept level, basic level and detail level. 


\section{Toward a Comprehensive Competency Modeling Framework}

\subsection{Competency Inherent Characteristic}

Competencies within an organization mainly can be categories in three groups, individual competency; enterprise competency and collaboration-oriented competency. The incorporation of the individual competency, enterprise's competencies and collaboration-oriented competency were referred as organizational competency [18] (see figure 1 from bottom to top), while a portion of the organizational competency which an enterprise decides to share within a network is entitled as sharing competency.

According to the U.S. Department of Labor, employment and training administration (ETA), individual competency, described as: [19] "the ability or capability of the individuals to apply the required set of skills, abilities and related knowledge to perform certain task successfully in a defined work setting". As a result of the ETA definition, Individual competencies denotes from the individuals of the organization. The second class of competencies is enterprise's competencies which refer to a set of competencies that constitute from enterprise facilities beside manufacturing and business knowledge [20]. This class of competencies captures information related to enterprise activists and resources and knowledge correlated to activity and resources [21].The third class of competency is collaboration-oriented competency, which is the organizations ability to cooperate and collaborate by working together towards achievement of a common goal [18]. Interaction and communication are highlighted as establishing the basis for shared understanding, which in turn is considered the basis for the creation of collaborative-oriented competency in a network [22].

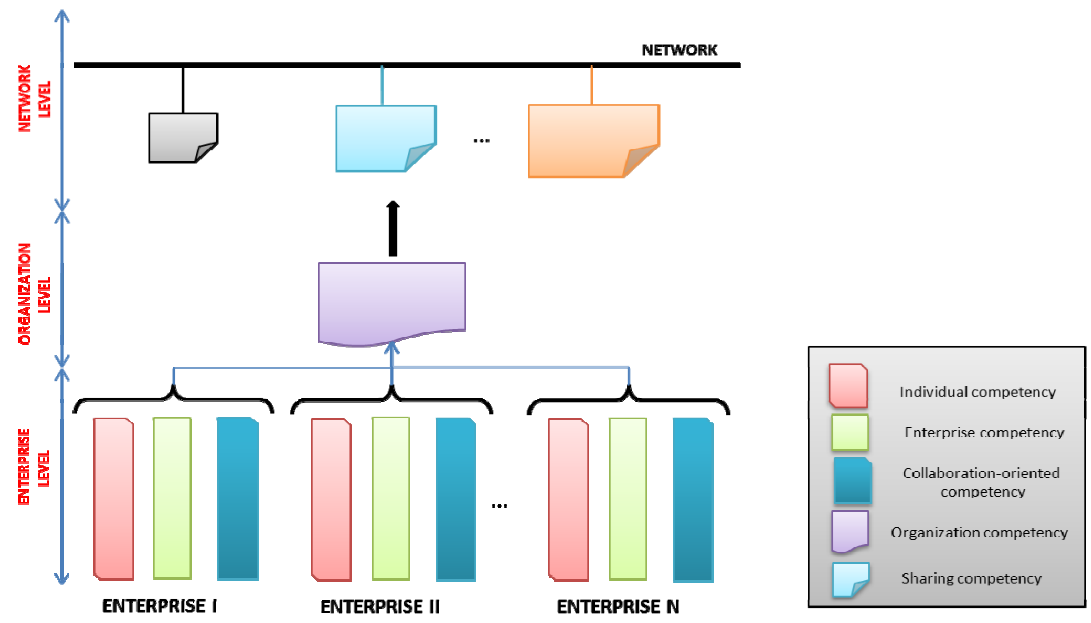

Fig. 1. Competency inherent characteristic 
Based on most recent categorization, the first defined dimension for competency modeling framework focuses on capturing competency inherent characteristics is represented by the vertical axis, labeled as "competency inherent characteristic". This perspective further includes three subspaces that comprehensively cover all the inherent characteristics of the organizational competency. The individual competencies of the organization (labeled "Individual competencies"); the enterprise competencies characteristics (labeled "Enterprise competencies competencies"), and collaboration-oriented competencies (labeled" Collective competencies").

\subsection{Modeling Viewpoint}

From the system theory points of view, competency is complex entities operating in a variety of environments having different purposes and internal/external manufacturing and business processes [23]. The existing competency models developed on two spaces namely; (a) managerial sciences and industrial engineering and, (b) information/knowledge managerial sciences (depicted in figure 3). These spaces although are related to each other, have different position in regards to the competency model, and thus the models defines in these views also differ.

On the other hand, the operating of competency models may occur at internal and/or external manufacturing business processes of an enterprise. [5] divide their researches on competency into intra-organization, inter-organization and network levels. Intra-organization oriented studies deal with the competencies within an organization. When the competencies are not bound to a single organization we talk of inter- organization level. Competencies for the static forms of cooperation among organizations that go beyond their boundaries could be subsumed under the term of supplier networks. Network level studies, consider, competencies need for creation of Virtual Enterprises as a network organization as well as competency as a tool for improve the VO performance.

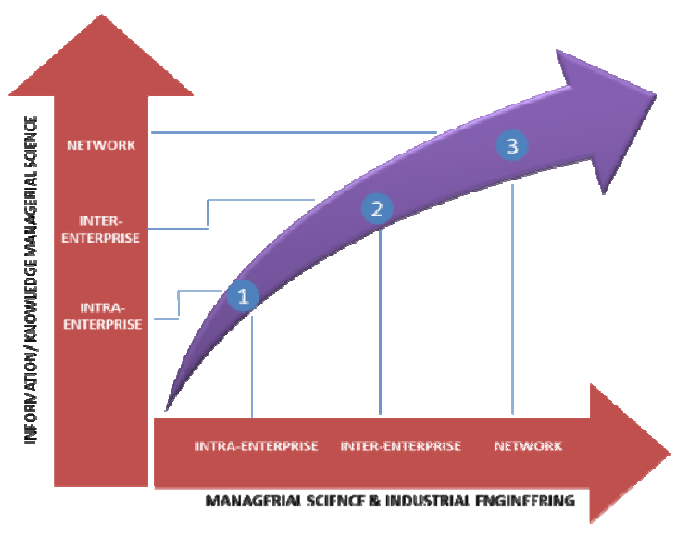

Fig. 2. Modeling perspectives for competency modeling 
The modeling perspective capture the modeling requirements and the diversity for internal and external manufacturing and business processes and for different purposes is be represented by the vertical axis, labeled as "modeling perspective".

The second defined modeling perspective addresses the competency modeling viewpoints as represented by horizontal axis on competency modeling framework. These viewpoints include "managerial science and industrial engineering" and "information/knowledge managerial sciences". Accordingly, competency at both viewpoints includes a variety of requirements and these requirements can be categorized into three different groups: intra- organization, competences within an organization; inter- organization, competencies beyond the boundary of the organization but for cooperation and network, competencies for the collaboration networks.

\subsection{Modeling Intents}

The contemporary organizational theories distinguish three hierarchical levels for organization management: organizational level, infrastructural level, and content level [24]. On the basis of such hierarchical system, it is possible to define three adequate levels in competency modeling processes. The third defined perspective is related to the different intents for the modeling of competency features, will be represented by the diagonal axis, and labeled as "modeling intents". This perspective addresses the three possible modeling stages for competency elements; from the organizational level, to the infrastructure level (e.g. using a specific modeling approach or theory), and finally to the content level.

Following the research practices in modeling, the three layers below are considered as follows:

- Organizational level: includes the most general concepts and related relationships, that is common to all competencies at the highest level, independent of the application domain.

- Infrastructure level: an intermediate level that includes more detailed models, focused on different classes of competencies.

- Content level: that represents models of concrete competencies.

\section{$4 \quad$ Proposed Modeling Frameworks}

In this section, the proposed modeling framework for organizational competency is presented. To begin with, the general modeling framework is given.

For the sake of consistency, the authors have named the suggested modeling framework, Comprehensive Organizational Competency Modeling Framework (COCMF). Figure 4 shows the developed modeling framework for the competencies. It can be argued that organizational competency models can be encapsulated into a process of three dimensions: Competency inherent characteristic, Modeling perspective and Modeling intent. COCMF explore the granularity of the competency with the purpose to systemize competency models which will be applicable for the 
transformation of an organization into a knowledge-based system [25], and its alignment with business goals and the range of other business management functions [26].

In this matrix, for the three subspaces of the individual competency, enterprise competency and collaboration-oriented competencies which forms the "competency inherent characteristic" dimension; their respective dimensions are depicted as different columns. Similarly, for the modeling perspective, each perspective is depicted as one row. The Modeling Intent constitutes the third dimension of the matrix, with its three respective subspaces of organizational level, infrastructure level and content level.

Each of 54 items within the COCMF possesses its own semantics and identifies the definite component of competency model, which integrates three dimensions: modeling perspective, inherent characteristic and modeling intents at the same level of elaboration. E. g., item 111 represents the integration of a competency model concerning three aspects of individual competency, intra-enterprise at managerial science points of view that are used at the organizational level. There are three, twodimensional subspaces of the COCMF, namely, E1, E2 and E3.

The subspace E1 "modeling perspective- competency inherent characteristic" defines models that are used to support modeling perspective at a definite inherent characteristic (individual, enterprise and collaboration oriented). The subspace E2 "modeling perspective-modeling intents" describes modeling perspectives and their interactions with the modeling intents. The subspace E3 "inherent characterizesmodeling intents" characterizes the competency in the way it is used at each level of competency modeling.

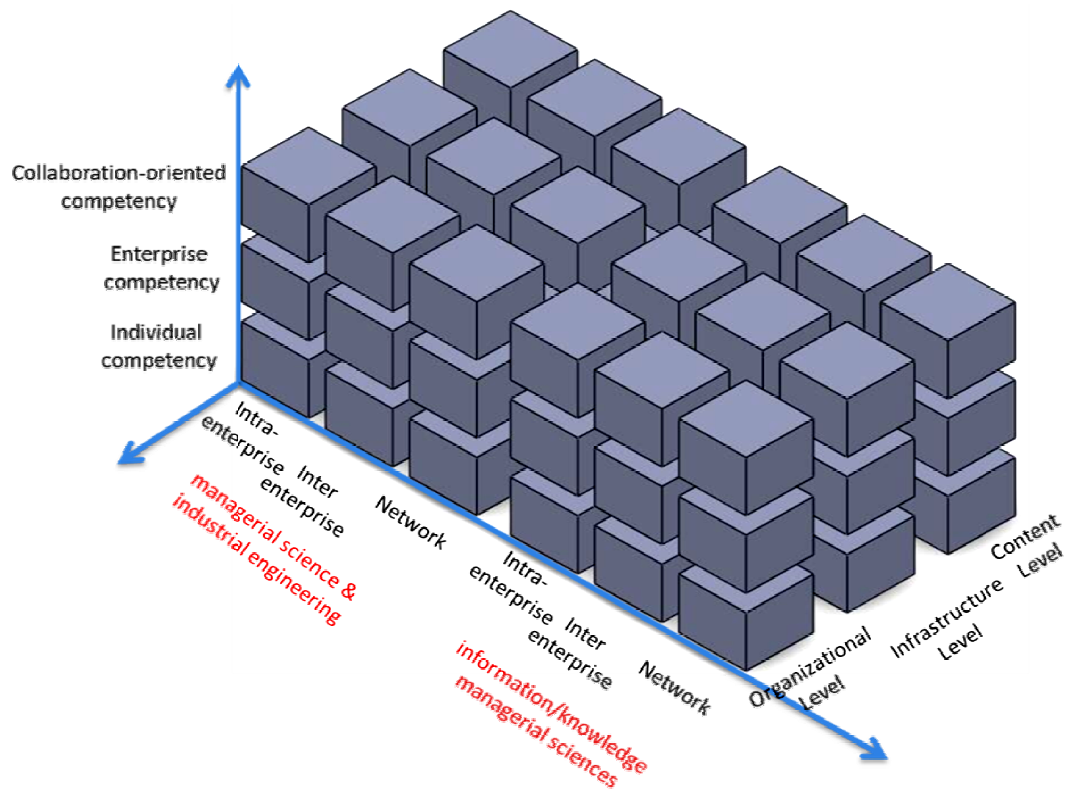

Fig. 3. A modeling framework for enterprise competency 
Our result of this study shows that although several related previous works have provided valuable contributions to the understanding of several aspects of this area, they are somewhat limited, when a more holistic modeling is pursued. This research suggests that organizational competency can be categorized into three groups, namely individual competency, enterprise competency and collaboration-oriented competency. For modeling each of these groups of competencies, it is essential that the modeling process have to be aligned with model developer purpose (Modeling perspective), so they exchange information, and operate based on the same segmentation model. Furthermore, competency have be model at different levels of abstraction (modeling intent). The main benefit of a competency modeling framework is enhancing decision making process for model developers. The main research limitation was; since the research is explorative in nature, therefore empirical data from similar and other research settings should be gathered to reinforce the validity of the findings. The major practical limitation of the COCMF is that a generic competency modeling approach is still needed for organizational competency modeling.

\section{Conclusions}

Establishment of a comprehensive modeling framework for organizational competency is a very important basis for the explanation of a generic competency modeling approach, a base element in the consolidation of existing knowledge in this area, tool for model developers for selecting appropriate competency models and a basis for its consistent further progress. In this way, as a contribution, a modeling framework for organizational competency considering multiple dimensions is proposed. The necessity and detail elements of each of the three dimensions on the proposed modeling framework, i.e. competency inherent characteristic, modeling perspective, and modeling intents are addressed. Finally, to benefit from the knowledge generated by other related research in this area, the most relevant other well-known competency models aspects and domains are evaluated with the three dimensions of the proposed modeling framework.

\section{References}

1. Prahalad, C.K., Hamel, G.: The core competence of corporation. Harvard Business Review, 79-91 (1990)

2. Boucher, X., Peillon, S., Burlat, P.: Towards a decision support for a collaborative increase of competencies within networks of firms. Research Report G2IEMSE, 600-609 (2005)

3. Mueller, E.: Production planning and operation in competence cell based networks. Production Planning \& Control 17(2), 99-112 (2006)

4. Saulius, G.: Enterprise knowledge modeling: domains and aspects. Baltic Journal on Sustainability 15(2), 281-293 (2009)

5. Ermilova, E., Afsarmanesh, H.: Competency modeling targeted on boosting configuration of virtual organizations. Production Planning \& Control: The Management of Operations, 103-118 (2010) 
6. Zoiopoulos, I.I., et al.: Identifying organizational competencies in project oriented companies: an evolutionary approach. In: Procs. 24th Annual ARCOM Conference, Cardiff, UK, September 1-3 (2008)

7. Bhamra, R., Dani, S., Bhamra, T.: Competence understanding and use in SMEs: a UK manufacturing perspective. International Journal of Production Research, 2729-2743 (2010)

8. Ermilova, E., Afsarmanesh, H.: Modelling and management of profiles and competencies in VBEs. International Journal of Intelligent Manufacturing 18(5), 561-586 (2007)

9. Vatankhah, R., Hashemipour, M., Guerra, D.: Toward a framework for intra-enterprise competency modeling. In: 2nd IEEE International Conference on Advanced in Computational Tools for Engineering Applications (ACTEA), Beirut (2012)

10. Camarinha-Matos, L.M., Afsarmanesh, H.: Towards a reference model for collaborative networked organizations. In: Shen, W. (ed.) Information Technology for Balanced Manufacturing Systems. IFIP, vol. 220, pp. 193-202. Springer, Boston (2006)

11. Rosemann, M., van der Aalst, W.M.P.: A configurable reference modelling language. Information Systems, 1-23 (2007)

12. Boucher, X., Bonjour, E., Grabot, B.: Formalisation and use of competencies for industrial performance optimisation: A survey. Computers in Industry, 98-117 (2007)

13. Javidan, M.: Core competence: What does it mean in practice? Long Range Planning, 60$71(1998)$

14. Ljungquist, U.: Core competency beyond identification: presentation of a model. Management Decision, 393-402 (2007)

15. Gilgeous, V., Parveen, K.: Core competency requirements for manufacturing effectiveness. Integrated Manufacturing Systems, 217-227 (2001)

16. Zhang, M.J., Lado, A.A.: Information systems and competitive advantage: a competency based view. Technovation, 147-156 (2001)

17. Walsh, S.T., Linton, J.D.: The Competence Pyramid: A Framework for Identifying and Analyzing Firm and Industry Competence. Technology Analysis \& Strategic Management, 165-177 (2001)

18. Kokko, N., Vartiainen, M., Lönnblad, J.: Individual and collective competences in virtual project organizations. The Electronic Journal for Virtual Organizations and Networks, 2852 (2007)

19. Competency, competency framework and competency model on line referencing, http: / / www. careeronestop.org/competencymodel / default.aspx (accessed February 11, 2011)

20. Kristianto, Y., Helo, P., Takala, J.: Manufacturing capabilities reconfiguration in manufacturing strategy for sustainable competitive advantage. International Journal of Operational Research, 82-101 (2011)

21. Blanch, R., Ferrer, I., Garcia-Romeu, M.L.: A model to build manufacturing process chains during embodiment design phases. International Journal of Advanced Manufacturing Technology, 421-432 (2012)

22. Paszkiewicza, Z., Picard, W.: Modelling Competences for Partner Selection in ServiceOriented Virtual Organization Breeding Environments. Journal of Computers \& Industrial Engineering, 1-17 (2011)

23. Vartiainen, M.: Mobile virtual work - concepts, outcomes and challenges. In: Mobile Virtual Work: A New Paradigm?, pp. 13-44. Springer, Heidelberg (2006)

24. Sormaz, D.: Distributed Modeling of Manufacturing Activities using Integrative Manufacturing Process Model. International Journal of Industrial Engineering and Management, 9-18 (2010)

25. Vichare, P., et al.: A Unified Manufacturing Resource Model for representing CNC machining systems. Robotics and Computer-Integrated Manufacturing, 999-1007 (2009) 